\title{
Los padres también se deprimen en el postparto: comprendiendo el fenómeno desde la voz de sus protagonistas
}

\author{
Alyson Morales \\ Universidad Alberto Hurtado, Santiago, Chile. \\ Email: a.moralesperalta@gmail.com \\ Anthea Catalán \\ Universidad Alberto Hurtado, Santiago, Chile. \\ Email: antheacatalanh@gmail.com \\ Francisca Pérez \\ Universidad Alberto Hurtado, Santiago, Chile. \\ Email: frperez@uahurtado.cl
}

\begin{abstract}
Resumen: Las investigaciones internacionales muestran una prevalencia de un $10.4 \%$ de depresión postparto paterna en la población general. En Chile el fenómeno no ha sido estudiado, por lo que nos propusimos explorar la presencia de sintomatología depresiva en padres chilenos durante el postparto, así como la particularidad de este fenómeno desde la subjetividad de los afectados. En este estudio exploratorio, transversal y cualitativo, se le realizaron entrevistas en profundidad a un subgrupo de padres que se situaron sobre el puntaje de corte de la Escala de Depresión Postparto de Edimburgo y/o el Inventario de Depresión de Beck, indagando en sus significados de paternidad y depresión. Entre los resultados destaca la no planificación del embarazo en todos los participantes, así como una paternidad asociada al rol proveedor. El concepto de depresión se asocia a sus manifestaciones más extremas. Si bien los padres presentan malestar subjetivo, no lo identifican como sintomatología depresiva, limitándose así la consulta espontánea y oportuna.
\end{abstract}

Palabras claves: Padres, sintomatología depresiva, postparto

\section{Fathers also get depressed in the postpartum: understanding the phenomenon from the voice of its protagonists}

\footnotetext{
Abstract: International research shows a prevalence of $10.4 \%$ of paternal postpartum depression in the general population. In Chile the phenomenon has not been studied, so we set out to explore the presence of depressive symptoms in Chilean parents during the postpartum period, as well as the particularity of this phenomenon from the subjectivity of those affected. In this exploratory, cross-
} 
sectional, qualitative study, in-depth interviews were conducted with a subgroup of parents who placed themselves on the Edinburgh Postpartum Depression Scale slice score and/or the Beck Depression Inventory, looking at their meanings of parenthood and depression. Among the results, the non-planning of pregnancy in all participants stands out, as well as paternity associated with the provider role. The concept of depression is associated with its most extreme manifestations. Although the parents present subjective discomfort, they do not identify it as depressive symptomatology, thus limiting spontaneous and timely consultation.

Keywords: Parents, depressive symptomatology, postpartum

\section{Os pais também ficam deprimidos no pós-parto: entendendo o fenômeno a partir da voz de seus protagonistas}

Resumo: Pesquisas internacionais mostram uma prevalência de 10,4\% de depressão pós-parto paterna na população geral. No Chile o fenômeno não tem sidoestudado, por isso partimos por explorar a presença de sintomas depressivos nos pais chilenos durante o pós-parto ea peculiaridade desse fenômeno a partir da subjetividade das pessoas afectadas. Nesta pesquisa exploratória, de corte transversal e qualitativaforam realizadas entrevistas em profundidade com um subconjunto de pais que situavam-se sobre o corte da Escala de depressão pós-parto de Edimburgo e/ou o inventário de depressão de Beck, investigando os seus significados de paternidade e depressão. Entre os resultados destaca-se o não planejamento da gravidez em todos os participantes, bem como uma paternidade associada ao papel do provedor. $\mathrm{O}$ conceito de depressão está associado às manifestações mais extremas. Embora os pais apresentem desconforto subjetivo, eles não o identificam como uma sintomatologia depressiva, limitando assim a consulta espontânea e oportuna.

Palavras-chave: pais, sintomatologia depressiva, pós-parto

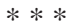

\section{Perinatalidad: cuando la demanda sobrepasa los recursos}

Además de la frecuente alegría y expectación que caracteriza a la etapa perinatal, esta implica una serie de ajustes y desafíos personales y familiares (Cierpka, Scholtes, Frey, \& Köhler, 2011; Epifanio, Genna, Luca, Roccella, \& Grutta, 2015; Stern, Bruschweiler-Stern, \& Freeland, 1998). Algunos de estos desafíos son asegurar la sobrevida del bebé, garantizar su desarrollo psíquico, generar una matriz de apoyo y balancear los distintos roles (madre/padre, trabajador/a, pareja, hija/o) (King, 2010; Stern, 1998). Se ha descrito que esta etapa constituye una crisis normativa en la vida de las personas (Frevert, Cierpka, \& Joraschky, 2008) existiendo la posibilidad de seguir creciendo y desarrollándose, pero también el peligro potencial de que la demanda sobrepase los recursos intrapsíquicos, interpersonales y contextuales existentes. En caso de ser así, es probable que aumenten significativamente los niveles de estrés, ansiedad y sintomatología depresiva en los miembros del subsistema parental, lo que incide negativamente a nivel personal, familiar y en el desarrollo de las/os niñas/os (Baldwin \& Kelly, 2015; Paulson, Bazemore, Goodman, \& Leiferman, 2016). 
LaOrganización Mundial de la Salud (OMS) ha enfatizado la importancia de evaluar la condición de salud mental materna en los controles de salud perinatales (WHO, 2016), considerando la robusta evidencia que señala que la sintomatología depresiva perinatal es común en la población femenina (10 - 20\%) (J. Goodman, 2004; O’Hara, 2009; Paulson et al., 2016), que sería más frecuente que en otras etapas del ciclo vital (Gavin et al., 2005), que las cifras se duplicarían en países en vías de desarrollo (Alvarado et al., 2000; Field, 2011) y que tendría consecuencias negativas en el desarrollo socioemocional y cognitivo de los/as niños/as (Coyl, Roggman, \& Newland, 2002; S. Goodman et al., 2011).

En Chile se realiza un tamizaje universal en la población femenina de depresión pre y postnatal, utilizando la Escala de Depresión Postparto de Edimburgo (EDPE), instrumento que ha sido validado en esta población (Alvarado, Guajardo, Rojas, \& Jadresic, 2012; Jadresic, Araya, \& Jara, 1995). Las estadísticas refieren que en nuestro país un 30\% de las mujeres gestantes presentan síntomas inespecíficos de depresión y/o ansiedad (Jadresic, Nguyen, \& Halbreich, 2007), y un 10\% depresión clínica (Jadresic, 2010), observándose además, que las mujeres que desarrollan un cuadro depresivo durante el embarazo tienen una mayor probabilidad de mantenerlo después del nacimiento del bebé (Alvarado et al., 2000).

\section{Acerca de la participación del padre}

En Chile, en relación a las tareas de cuidado infantil y la corresponsabilidad en la crianza, desde los noventa se han presentado propuestas legislativas y programáticas que incorporan a los hombres y a otras personas en tareas de cuidado de las/os niñas/os, detrás de las cuales hay un discurso político que impulsa cambios culturales en la redistribución de los roles de cuidado y crianza de los/as hijos/as (Casas \& Valenzuela, 2012). No obstante, algunos estudios muestran la existencia de tensiones e inconsistencias entre el nivel discursivo y el nivel conductual, la política y los marcos legislativos (Olavarria \& Marquéz, 2004; X. Valdés \& Godoy, 2008). Sin ir más lejos, las condiciones de salud mental del padre no son incluidas en el screening universal que sí se hace con las mujeres, a pesar de la creciente evidencia que señala que la sintomatología depresiva perinatal paterna también es un fenómeno recurrente y que tiene impacto en la salud mental del sistema familiar (A Faisal-Cury \& Menezes, 2007; Condon, Boyce, \& Corkindale, 2004; Escribe-Agüir, Gonzalez-Galarzo, Barona-Vilar, \& Artazcoz, 2008; J. Goodman, 2004).

Una revisión de la literatura que consideró estudios con revisión de pares publicados entre enero del año 2005 y enero del 2016 mostró que la mayoría de los estudios que han evaluado sintomatología depresiva paterna durante el primer año postparto han sido llevados a cabo en Europa, son de carácter longitudinal y utilizaron escalas de autoevaluación. Las cifras encontradas fluctuaban entre un $1.8 \%$ y un $47 \%$, lo que podría explicarse por la debilidad metodológica de los estudios, considerando que muchas de las escalas aplicadas no estaban validadas, así como por las 
diferencias culturales en torno a la masculinidad, paternidad y depresión, considerando que los países que mostraban cifras más bajas adherían predominantemente a ideologías de género tradicionales (Pérez, Brahm, et al., 2017).

Estudios correlacionales han encontrado una relación positiva entre la sintomatología depresiva perinatal paterna y materna, señalando incluso que esta última sería el principal predictor de la sintomatología depresiva paterna (J. Goodman, 2004; Paulson \& Bazemore, 2010). Asimismo se sabe que la sintomatología depresiva perinatal paterna impacta el desarrollo emocional y conductual en bebés y niñas/os entre 3 y 5 años, afecta la dinámica familiar, la satisfacción marital y la economía de países industrializados (Paulson, Keefe, \& Leiferman, 2009; Ramchandani, Stein, Evans, O’Connor, \& Team, 2005). Por su lado, estudios cualitativos han mostrado que la sintomatología depresiva masculina tendría manifestaciones específicas, como aumento de la hostilidad, conflicto y enojo, más que expresión de tristeza (Condon et al., 2004). Los padres tienden a ser más evitativos y a desarrollar actividades que los mantengan al margen de la crianza, como aumentar la carga de trabajo, el deporte, el sexo o los juegos de azar, así como la automedicación o el consumo de sustancias como el alcohol (Veskrna, 2013).

En Chile no contamos con evaluciones del fenómeno, por lo que lapresente investigación, de carácter exploratoria, da cuenta de los resultados de las entrevistas en profundidad que se le realizó a un subgrupo de padres que presentó sintomatología depresiva durante el postparto, de manera de conocer sus características y manifestaciones desde una aproximación subjetiva. Las preguntas que guiaron este estudio fueron: ¿Cómo viven la paternidad un grupo de padres que han presentado sintomatología depresiva durante el postparto?¿Ha habido desafíos de la paternidad que los hayan hecho sentir sobrepasados? ¿Cuáles son los significados de depresión que tienen estos padres?

\section{Metodología}

\section{Diseño}

Investigación de tipo exploratoria, transversal y cualitativa, ya que se busca obtener la comprensión de los fenómenos desde la subjetividad de sus protagonistas.

\section{Muestreo y Participantes}

El muestreo fue intencionado, ya que se incluyeron aquellos padres que se situaron sobre el puntaje de corte en la Escala de Depresión Postparto de Edimburgo (EDPE) y/o en el Inventario de Depresión de Beck (BDII)(Beck, Ward, Meldelson, Mock, \& Erlbaugh, 1961). Además estuvo situado en el contexto del Servicio Nacional Metropolitano Occidente de Salud, 
específicamente usuarios del Hospital San Juan de Dios (HSJD), que atiende a población de las comunas de Pudahuel, Renca, Cerro Navia, Quinta Normal y Lo Prado en Santiago de Chile.

\section{Procedimiento}

El contacto se realizó inicialmente en el HSJD en el área de Ginecología, invitando a participar a los padres y madres durante la hospitalización de postparto. En esa instancia se recabaron los datos de ambos y se firmó el consentimiento informado. Dos meses después las parejas fueron vueltas a contactar, instancia en la que se aplicó a 65 padres, ya sea vía web o telefónica, la EDPE, el BDI-I y un cuestionario sociodemográfico. Los padres que se situaron sobre el puntaje de corte en la EDPE (10 puntos para población femenina) (Jadresic et al., 1995) y/o en el BDI (13/14 puntos) (C. Valdés et al., 2017) fueron invitados a participar de una entrevista en profundidad, la cual se realizó en el domicilio de los participantes, con una duración promedio de 60 minutos.

\section{Técnica de la producción de datos}

Entrevistas en profundidad. La entrevista es una teìcnica cualitativa que permite rescatar la subjetividad de los participantes, en este caso, los significados de paternidad y de sintomatologiìa depresiva perinatal que emerjan en los padres. Estas contaron con un guioìn temaìtico que se elaboró en funcioìn de los objetivos del estudio, siendo utilizado de forma muy general y flexible, de manera que tanto los entrevistados como el entrevistador pudieran desarrollar temas inesperados. Siguiendo el caraìcter iterativo de la investigacioìn cualitativa, ademaìs de ser piloteados, los guiones de entrevistas desarrollados fueron ajustados como consecuencia del análisis de los emergentes. Las entrevistas fueron grabadas en audio y transcritas para su posterior anaìlisis.

Escala de Depresioìn Postparto de Edimburgo (EDPE) (Cox, Holden, \& Sagovsky, 1987). Escala auto-administrada que da cuenta de la presencia de sintomatologiìa depresiva. La escala tiene un valor maìximo de 30 puntos, de los cuales una puntuacioìn de 10 o maìs indica una posible depresioìn, de diversa gravedad (Eberhard-Gran, Eskild, Tambs, Opiordsmoen, \& Samuelsen, 2001). Fue validada en Chile en mujeres durante el embarazo mostrando una estructura unifactorial, una alta consistencia interna (.914) y una alta correlacioìn con el BDI-I $(\mathrm{r}=.85, \mathrm{p}<.001)$ (Alvarado et al., 2012) y en el postparto mostrando una buena consistencia interna ( $\alpha$ $=.77$ ) (Jadresic et al., 1995) logrando su mayor sensibilidad con un punto de corte 9/10 (Eberhard-Gran et al., 2001). Los estudios en poblacioìn masculina han mostrado una buena sensibilidad y especificidad del instrumento, sin embargo, los resultados no son conclusivos (Edmonson, Psychogiou, Vlachos, Netsi, \& Ramchandani, 2010; Lai, Tang, Lee, Yip, \& Chung, 2010; Massoudi, Hwang, \& Wickberg, 2013; Matthey, Barnett, Kavanagh, \& Howie, 2001; Tran, Tran, \& Fisher, 2012). En este estudio la escala mostró una buena aceptabilidad y una alta correlacioìn con el BDI-I $(r=.84, p<.01)$ (Pérez, Catalán, et al., 2017). 
Inventario para la Depresión de Beck (BDI-I) (Beck, Ward, Meldelson, Mock, \& Erlbaugh, 1961). Cuestionario de auto reporte que consta de 21 ítems y evalúa la sintomatología depresiva actual en adultos. En esta prueba el sujeto debe elegir, entre un conjunto de cuatro alternativas ordenadas de menor a mayor gravedad, la frase que mejor describe su estado durante la última semana. Cada ítem puede ser valorado de 0 a 3 puntos, pudiendo obtener un puntaje total que varía entre 0 y 63 . Puntajes mayores indican mayor sintomatología depresiva y se definen 4 categorías de depresión: mínima 0-9, leve 10-18, moderada 19-29 y severa 30-63. Los análisis de fiabilidad son adecuados, obteniendo la versión española aplicada en pacientes con trastornos psicológicos un valor $\alpha=.90$ (Vázquez \& Sanz, 1999). En la versión chilena, en proceso de validación por el Núcleo Milenio Psicoterapia y Cambio en Depresión, se reporta un valor $\alpha=.92$ en población clínica (C. Valdés et al., 2017). En la muestra actual, se encontró un $\alpha=.90$ para la muestra total, siendo $\alpha=.86$ en la muestra de padres y de $\alpha=.91$ para las madres, lo que se considera adecuado.

\section{Análisis de datos}

Para dar cuenta de los objetivos del estudio, la información entregada por los instrumentos cuantitativos fue analizada a través de estadística descriptiva, de manera de poder caracterizar a la muestra en relación a sus antecedentes sociodemográficos y la presencia/ausencia de sintomatología depresiva perinatal.

Los datos que emergieron de las entrevistas se abordaron desde la perspectiva del análisis dialógico del discurso donde el foco se ubicó en el relato mismo de los participantes, el modo en que este fue articulado y desde dónde estaban hablando al momento de dar cuenta de su experiencia. En ese sentido, la atención estuvo puesta en el contenido, pero también en lo procedimental (ritmos, pausas, silencios, quiebres, etc). Cada caso fue analizado de manera singular, donde se realizaron informes de caso en los cuales se detallaron ciertas concepciones en relación a la paternidad y la depresión con otros factores relevantes como el trabajo, la pareja, el bebé, lospropios padres, entre otros. En dicha instancia se reflexionó con el equipo, compuesto por tres psicólogas (todas mujeres), en torno a la información presentada en cada caso, discutiendo los emergentes y añadiendo nueva información relevante en los guiones de entrevista. Finalmente se realizó un análisis transversal que permitió comparar, distinguir, describir y aunar criterios en relación a los diferentes casos abordados y los emergentes de cada uno de ellos. A través de este procedimiento fue posible contrastar y organizar los resultados en torno a categorías generales transversales que permitieron la construcción de los resultados.

\section{Caracterización de los participantes}

La muestra final obtenida constituye un grupo bastante homogéneo de varones con niveles medios de educación formal y actualmente insertos 
laboralmente, la mayoría en relación de dependencia y en trabajos full- time. Los sujetos participantes del estudio fueron 65 varones heterosexuales mayores de 18 años que tenían por lo menos un hijo/a de menos de un año de edad y que convivían actualmente con la pareja con quien comparten la crianza. Un 18.5\% (12 sujetos), puntuaron sobre el puntaje de corte en la EDPE y un 10.5\% (6 sujetos) sobre el puntaje de corte en el BDI. De este total de sujetos que presentaban sintomatología depresiva, siete fueron entrevistados en sus domicilios.

La edad de los participantes entrevistados estuvo comprendida entre los 22 y los 35 años ( $M=29.14$; DS =4.26). En cuanto al nivel de estudios la mayoría había alcanzado la enseñanza media completa. Respecto a la situación de pareja, un tercio de los padres eran solteros y dos tercios casados. Respecto de la ocupación actual, la construcción, el comercio, el transporte y las actividades de servicio de alojamiento y comidas fueron las ramas de productividad económica reportadas. En cuanto a las condiciones laborales la mayor parte de los entrevistados se encontraba con contrato de honorarios, jornada completa.

\section{Resultados}

El análisis de los resultados se realizó considerando las características de los participantes anteriormente descritas sin pretender generalizar los mismos por fuera de este grupo en particular, sino que orientar futuras líneas de indagación, que permitan profundizar en las creencias y prácticas en torno a la paternidad y la depresión, y establecer eventualmente diferencias con varones de otros estratos socio-económicos. El análisis del material obtenido se articuló en torno a dos ejes principales: 1) experiencias y desafíos de la paternidad y 2) significados y experiencias de depresión.

\section{Eje 1: experiencias y desafíos de la paternidad}

El eje transversal de esta investigación se construye a partir del relato que hacen los padres entrevistados de su experiencia de paternidad, enfatizando aquellos aspectos que han resultados desafiantes y que eventualmente pudiesen estar ligados a la sintomatología depresiva que presentan.

\section{La irrupción de una paternidad “no planificada”}

Un primer elemento común que se presenta en la totalidad de los casos entrevistados es la no planificación del embarazo. La noticia del embarazo y la paternidad irrumpe en la vida de los entrevistados como un factor inesperado, apresurando e imponiendo la necesidad de asumir este nuevo rol de forma positiva.

"Eh no, no estaba planeado, o estaba pensado, ja... eh, se dio y bueno se asumió también, se asumió en el momento” (E1).

"No lo teníamos esperado, porque igual ella tomaba... estaba en tratamiento y de repente...”(E2).“No fue esperado y ella se lo tomó 
súper mal” (E3).

"Fue extraño igual no era como intencional, no esperábamos tener un bebé, pero cuando supimos fue bacán” (E4).

“En realidad no fue planificado, o sea, salió no más” (E5).

"Entonces fue como planeado, pero no todavía, teníamos pensado hacerlo un poquito más adelante” (E6).

"Al principio no lo podía creer. Pero no, ya después asumí y esperar po, ehm... lo que sea” (E7).

Asumir este nuevo rol se asocia en el discurso a la necesidad de “madurar”, es decir, la necesidad de dejar aspectos más infantiles o adolescentes de la vida que se llevaba, como la dependencia económica, las salidas con los amigos y el carrete. Si bien esta necesidad es compartida por todos los entrevistados, para algunos este proceso es vivido de forma natural y coherente con sus experiencias previas, en especial para aquellos hombres que ya tenían hijos/as anteriores, mientras que para otros este mandato es vivido con mucho conflicto.

"Bueno fue distinto por el tema de que eh... yo fui papá a los 21 años y ahora fui papá a los 27 años, entonces entre ese lapso de tiempo obviamente uno aprende y madura más, cambia un poco la perspectiva de las cosas, de cómo uno lo asume, cómo lo ve. Entonces en ese sentido yo fui más maduro, disfruté un poco más... “ (E1).

"Sí, o sea igual con el Matías fue el primero y él me hizo aterrizar porque el Matías nació cuando tenía como 24, y en ese tiempo uno anda en la onda carrete y toda la cuestión” (E5).

"Para mí como que no ha cambiado nada... porque igual sigo haciendo las cosas como que no... como que si yo fuera soltero, pero eso tengo que cambiarlo yo po, porque igual está mal, porque igual como que no pienso en nada de repente, como que creo que no tengo pareja, no tengo hijo, no tengo nada... y eso” (E3).

\section{Rol paterno: ideas, prácticas y sensaciones}

Asumir el rol de padres se asocia a la necesidad de constituirse en una figura de apoyo y sostén para su pareja e hijo/a, ya que se considera que es la mujer que se lleva una mayor sobrecarga durante el embarazo, parto y postparto.

“(...) ser un apoyo en el momento a nuestra pareja, a la mujer, para que ella no esté sola y estar involucrado un poco más con el tema de lo que es el tener un hijo (...) La mujer igual se lleva harto peso... eh... desde que parte su embarazo hacia adelante... yo creo que eso puede ser un poco... entenderla” (E1).

Todos los entrevistados comparten la idea de que el padre debe ser una figura de apoyo y sostén para que su pareja esté lo mejor posible durante el embarazo, parto y postparto, ya que se considera que es ella quien se encuentra en una posición de mayor vulnerabilidad y dependen- 
cia. Si bien durante el embarazo hay mayor heterogeneidad en los significados y prácticas de lo que es "ser un apoyo", en el parto hay unanimidad en la relevancia que se le otorga a la propia participación en este. Todos los padres refieren haber estado presentes durante del parto, lo que se ajusta a las nuevas políticas públicas fomentadas por el Sistema de Protección Social Chile Crece Contigo. Todos los entrevistados consideran que era muy importante estar presentes en ese momento, para acompañar a su pareja y recibir a su hijo/a, sin embargo, al encontrarse en el escenario in situ las reacciones fueron diversas. Para algunos padres, en especial para los primerizos que no habían tenido ningún tipo de preparación previa, el presenciar ese momento resultó chocante y, en un par de casos, los padres reconocen haber deseado escapar de la situación.

“Ahí le digo a la enfermera que hay otra cosa más que no sé qué es, entonces así le indiqué que fuera a ver y cuando volvimos estaba la cabeza afuera, ¡estaba saliendo! Y yo me quería arrancar (...) pasó que inmediatamente entró la matrona y toda la gente y decidí salir porque pensaba que quizás a lo mejor estoy estorbando aquí, y me quedé en el pasillo y la enfermera después me agarró y me dijo que entrara porque tenía que estar ahí en todo eso” (E5).

"Y ahí yo llegué... y cuando llegué... llegué a cierta hora y ya... ella ya estaba lista pa que saliera la guagua... si yo cuando entré ya tenía la cabecita afuera y yo me sorprendí porque así como que... es como que es fuerte igual po...” (E3).

Una vez que nace el bebé, todos los padres coinciden en que es la madre la principal responsable de la crianza y el bienestar del niño/a, teniendo ellos un rol secundario.

"El papá... el papá no lleva tanta responsabilidad, porque en realidad él... osea más que nada es de que ella esté... la pareja esté bien, de que ella esté tranquila, esté... que no le falte nada, más que nada yo creo eso" (E1).

En el discurso de los padres son las madres y las abuelas las expertas en los cuidados del bebé, sintiendo que la propia participación más que ser una ayuda es un estorbo. Para algunos esta situación es coherente con sus ideas de paternidad (roles tradicionales), pero para otros que genuinamente tienen ideas más igualitarias de cuidado, esta situación genera malestar.

"No tengo ese rol de padre así participativo... o sea como que no que... es que donde son muy chiquititos como que me da cosa igual... con mi hija tampoco participé mucho... yo me daba cosa mudarla... de bañarla también... y eso no sé si será malo, pero no me gusta” (E3).

"Es que yo la trato de ayudar pero ella nunca me... me dice, bien; siempre me está retando (...) siempre me dice, es que "ah no, es que no sabí cambiarle los pañales”, "ah, es que no sabí esto, que no sabí esto otro", "que estai con el teléfono en la mano"; me reta por todo" (E2). 
Las responsabilidades que son consideradas propias del padre se ajustan a una perspectiva de género tradicional, donde lo que predomina es el rol proveedor, los aspectos prácticos y materiales de la vida en familia. Un ejemplo de ello, se refleja en la siguiente cita, cuando a uno de los padres se le pregunta respecto al beneficio de posnatal.

"Sí, osea me tomé los días del posnatal, hice los trámites que tenía que hacer... la inscribí, qué más? Hice todos los trámites... la inscribí en mi seguro, eh... hice todos los papeles relacionados con recién nacido" (E1).

En relación a la crianza propiamente tal, el principal rol que destaca es el de “educador”, donde en proyecciones futuras aparecen ideas asociadas la transmisión de normas, valores, herramientas y de una educación formal que les permitan enfrentarse a el mundo con autonomía y responsabilidad.

“Tratar de enseñarle, inculcarle bien las cosas como son de la vida, eh... enseñarle que hay gente que es mala (...), como también hay gente que igual la apoya (...) tratar de inculcarles mucho eso, el cuidado, con las personas que se juntan” (E1).

"O sea siempre siendo responsable (...) no me gusta que mientan, y eso le muestro a los niños, y la mentira chica es mentira no más, como desde chicos les intento decir eso” (E5).

\section{Proveer: el principal mandato del mundo masculino}

Para todos los entrevistados el rol paterno continúa estando predominantemente ligado a su función proveedora, la que en la mayoría de los casos aparece como una exigencia que es exclusiva del hombre. De esta forma, la etiqueta de sostenedor económico cobra gran relevancia en la propia definición, la cual si bien los moviliza hacia la búsqueda de mejores oportunidades laborales, también les genera mucha presión, miedo a no lograr llegar a fin de mes y frustración al sentir que a pesar de los esfuerzos no se logra lo esperado.

"Bueno harta responsabilidad igual porque si yo dejara de trabajar no hay sustento... entonces sí o sí tengo que trabajar, ser más esforzado quizás” (E1).

"Estoy feliz e igual me pareció una bendición pero como lo vamos hacer después, eso me angustia un poquito, como que... mi hija va a crecer ¿cachai? (...) no sé se viene difícil y no podemos estar viviendo a cuesta de nuestras familias aunque sean súper buena gente” (E4). "O sea discutimos por cosas que de repente faltan, faltan cosas de repente y uno arrienda acá, ahora sacábamos cuentas y andábamos cortos, entonces ya no soy el mismo de antes que uno antes no tenía más obligaciones y uno pasaba la tarjeta a los hijos, pero ahora estoy tratando de buscar otro trabajo mejor, para que podamos vivir mejor" (E7). 
Es importante añadir que en todos los casos nos encontramos frente a padres que cumplen jornadas laborales completas de 45 horas o incluso más, pero aún así en muchos casos no logran obtener un salario que les garantice poder solventar las necesidades básicas.

\section{Eje 2: significados y experiencias de depresión}

Uno de los elementos interesantes a indagar fueron las nociones y experiencias de depresión que tenían los entrevistados, considerando que todos presentaban sintomatología depresiva en una o ambas escalas aplicadas. En todos los casos aparecen definiciones extremas del diagnóstico, principalmente vinculadas a la ideación suicida activa, por lo cual se torna muy difícil poder reconocer la propia experiencia depresiva.

\section{Significados de depresión: la idea del túnel sin salida}

En la mayoría de los casos la depresión se asocia a la idea de no querer vivir, ver todo negro, querer hacerse daño o querer dañar a otra persona.

“Así depresión creo que es cuando uno se quiera matar... eso es lo único que pienso que puede ser... que no le encuentra salida... eh... como solución a sus problemas y lo único que decide es eliminarse... o que se ahogan en un vaso de agua" (E3).

"La depresión como lo entiendo yo es ver todo negro, no ver ninguna salida” (E5).

"Me imagino la depresión como una persona que no quiere... que ve todo negro, que se siente mal, que... quiere matarse, que quiere... no sé po, hacerle daño a otra persona quizás o hacerse daño a sí misma... yo encuentro que eso es como... no sé, nunca lo eh... nunca lo he visto en un hombre” (E1).

La depresión por muchos de los entrevistados se asocia a la imposibilidad de encontrar solución a los problemas, un fracaso en la agencia personal y por ende en la masculinidad.

"El orgullo de ser varón tiene que ver con no dar tu brazo a torcer" (E7).

"Y resulta de que eh... dije yo tengo que salir solo. No me la puede ganar ésta hueá” (E2).

\section{Vivencias de depresión}

Reconocer experiencias de depresión se vuelve amenazante, ya que atenta contra las ideas que se comparten de paternidad y masculinidad, principalmente la agencia y el sostén. Sin embargo, en el relato de los entrevistados aparecen vivencias que dan cuenta de la sintomatología depresiva, destacando en el plano emocional la irritabilidad, la tristeza, la frustración, el miedo y la angustia. 
“Ahí yo me desquitaba con la demás gente. Mi suegro me hablaba y yo decía ¡Qué!, mi mamá mehablaba y la quería mandar a la chucha. De repente en la pega” (E6).

Al poner estas vivencias en palabras uno de los entrevistados se cuestionó durante la entrevista si tendría o no depresión.

“No, no sé, a lo mejor yo tengo depresión y no me he dado cuenta, pero ando enojado, me enojo, llego aquí y es como llegar no a una casa, es como llegar a un lugar con puros problemas, puras deudas, te llenan los papeles de deudas y cosas, sí, conozco personas, han llegado al suicidio, a la droga, al alcoholismo, delincuentes” (E7).

A otro entrevistado, la lejanía física de su bebé recién nacido, le generó una fuerte ansiedad de separación, tristeza, disminución del apetito y desmotivación.

“A mí además me empezó esto porque; estuve dos semanas acompañándola, en el nacimiento de mi hija, y después cuando estaba esto me fui llorando, lloraba todos los días, lloraba... Y en cambio, yo pesaba noventa y siete kilos y ahora estoy pesando ochenta y uno (...) Dejé de comer, no tenía ganas de trabajar, en cambio los jefes me decían: ¿Qué te pasa que no estai rindiendo el... como tú trabajas?. Y yo les explicaba que echaba de menos a mi hija” (E2).

Como se esbozo anteriormente, un fuerte estresor y generador de angustia común para muchos padres es la presión que sienten por sostener económicamente a su familia, en especial al pensar en el futuro.

"Yo tengo una tendencia como a desanimarme o asustarme en verdad, por lo que decía del futuro es terrible angustiante, y siempre me ha pasado, mm pero ahora, me pasa más, por todo, la pega, no séel futuro, pero lo que me saca de eso es ver a mis dos mujeres” (E4). "El miedo más grande que tengo en la vida es que no me dé para darle las cosas que ella necesita. (...), y así y todo no me da, y para mí llegar a fin de mes es un trauma, me empiezo a ver que para qué esto, cómo saco para esto, que cómo lo hago para tenerle para sus leches, sus cosas, así son cosas que me tienen frustrado....” (E6).

En el plano conductual aparecen comportamientos que buscan mitigar las emociones anteriormente mencionadas, ya sea por la vía de la canalización o evitación de las mismas. El gimnasio o el deporte es una opción que buscan los padres como una vía de escape o de posibilidad en la cual encuentran pueden mantener un espacio personal, fuera de lo que contempla su vida familiar o su rol paternal, pero también las salidas con amigos y más específicamente el consumo de sustancias, es también una alternativa entre quienes fueron entrevistados. 
"Hago cosas que... no debería hacerlo pero igual me porto como mal... en el sentido que... igual como que me da cosa decirlo pero tomo y me drogo... hasta ahora... y me gasto dinero que me serviría pa' otras cosas" (E3).

Es importante señalar que un entrevistado señaló haber presentado ideación suicida, pero es importante destacar que esta ideación no se asocia a la paternidad propiamente tal, sino que a un cuadro depresivo de base no tratado, consumo de sustancias y conflictos con la pareja.

"Porque como que conmigo ha pasado muchas penas po... o... eso yo siento, que ha pasado muchas cosas conmigo que son feas y que antes no las pasaba... y que ella me lo dice po y yo igual me siento mal y eso me da rabia po... de repente así pienso que... así pienso tonteras po, porque digo que mejor que yo no existiera y todo va a estar bien... pero si yo no existo, ellos no van a... no sé... me imagino puras cosas tontas, son medias fomes las cosas que pienso” (E3).

Es importante señalar que algunos de los entrevistados, al preguntarles por experiencias adversas en su familia de origen señalan haber sufrido maltratos y negligencia en los cuidados, así como haber vivido situaciones de precariedad económica.

“Cuando chico mi papá y mi mamá tuvieron mucho drama ... mi papá a veces se iba y no volvía... habían muchas separaciones entre medio" (E1).

"Pero igual siempre dije que yo no quería ser como fue mi papá como fue con nosotros al principio. A mi papá no le importaba nada” (E6). "Nunca nos faltaron cosas para comer en la casa pero el trato fue malo. A ver le cuento. Como hijo cuando nosotros estábamos chicos, nos pegaba mucho mi papá. A mi mamá también, a mi hermano; éramos dos hermanos. A mi mamá le pegaba, a mi mamá no la dejaba salir a la calle. A nosotros, llegábamos del colegio nos mandaba a trabajar, lo cual, teníamos que arar la tierra nosotros con dos caballos” (E2).

"Eh que me viera el tomar igual po... pero se me ha hecho muy difícil si, sobre todo con la droga... ahí no me gustaría que ellos me vieran así... mal... o que... o de... cuando ellos se portan mal de pegarles así (...) capaz que a mí no me dieron amor también po, capaz que mi papá también era así... yo me imagino... no sé” (E3).

Estas experiencias adversas vividas en la infancia se asocian por los mismos entrevistados, en algunos de manera más directa, en otros de forma más tangencial, con los miedos e inseguridades actuales, que se reactualizan con el nacimiento del propio bebé. Muchos padres tienen la intención de hacerlo completamente distinto y poder reparar con sus hijos/as lo que tuvieron que vivir de niños/as, sin embargo, el temor y la frustración aumenta cuando sienten que no son capaces. 


\section{Discusión}

El foco del presente estudio fue escuchar las voces de padres que presentaron sintomatología depresiva durante el postparto, relevando así el hecho que no sólo las madres están vulnerables a presentar dificultades socioemocionales en esta etapa. Si bien, es importante destacar que la aproximación exploratoria y las características específicas del grupo entrevistado no permite generalizar las conclusiones, los resultados muestran que para este grupo de padres hay muchas ideas y vivencias compartidas en relación a la paternidad y a la sintomatología depresiva.

Lo primero que destaca es cómo la paternidad irrumpe en la vida de los entrevistados, sin que haya habido una planificación previa, noticia que se asumió con mayores o menores niveles de ambivalencia. La incapacidad de los entrevistados para planificar su paternidad hace que nos cuestionemos cuál es el acceso a información que han tenido estos padres, si han tenido o no educación sexual o qué tipo de vivencias relacionales han tenido que los hace vivir su vida sexual y de pareja de forma tan disociada.

Para la mayoría los entrevistados el rol paterno se inscribe en una ideología tradicional de roles de género, donde la madre es la principal encargada del bienestar y la crianza del bebé, mientras que la función del padre es principalmente la de proveer. Esto daría cuenta que pese a los cambios socioculturales y económicos que han impulsado la reorganización familiar (X. Valdés \& Godoy, 2008), en el imaginario y las prácticas colectivas los cuidados y la crianza continúan siendo considerados una tarea predominantemente femenina (Herrera \& Pavicevic, 2016; Miller, 2017). La mayoría de los padres no se refiere al vínculo particular que han creado con su bebé, ni a su desempeño en los cuidados de éste, refiriendo que su rol en este ámbito es secundario, e incluso algunos refieren vivenciar su participación como un estorbo. La paternidad durante el primer año de vida del bebé consiste más bien en apoyar a la madre y en proveer, y al proyectar la paternidad en el tiempo aparece el rol "educador", donde adquiere relevancia el hecho de traspasar a la siguiente generación normas y valores, que les permitan vivir autónoma y responsablemente.

Es importante destacar que para la mayoría de los casos el proveer económicamente aparece como una exigencia que es exclusiva del hombre, lo cual si bien los moviliza hacia la búsqueda de mejores oportunidades laborales, también es fuente de mucha presión y frustración. El futuro y la posibilidad de sostener económicamente a una familia, opera como el principal estresor a la hora de pensar los síntomas depresivos que están manifestando los padres, donde la sensación de "no llegar a fin de mes" a pesar de los esfuerzos que se realizan, sobrepasa a estos padres, quienes encuentran pocos espacios para desplegar y canalizar este malestar al interior de la vida familiar. Es así como muchos de ellos mantienen prácticas y vínculos que les permiten evadir el malestar, principalmente el deporte y/o "el carrete”. 
En la mayoría de los casos, el malestar que se pesquisa a partir de los cuestionarios y que corroboran las entrevistas en profundidad, no se asocia al diagnóstico de depresión ni a la posibilidad de presentar sintomatología depresiva, ya que la mayoría de los entrevistados asocia este concepto a sus manifestaciones más severas, como el suicidio. De esta forma, es muy difícil que los entrevistados puedan llegar a consultar espontánea y oportunamente, ya que el malestar no se asocia a la posibilidad de presentar un cuadro anímico. Sin embargo, cuando aplicamos las escalas que evalúan sintomatología depresiva, los padres reconocen y se identifican con varios de los síntomas de los instrumentoscomo el sentirse abrumados/presionados, la culpa, la disminución de la capacidad de goce, la irritabilidad, el cansancio, entre otros. Si bien, es importante considerar que al tratarse de un estudio transversal, no podemos asegurar que la sintomatología depresiva que presentan los padres haya sido gatillada específicamente por la experiencia de la paternidad, por lo que muestra la literatura, independiente del gatillante, sabemos que la presencia de sintomatología depresiva en esta etapa tiene efectos adversos en el funcionamiento familiar y el desarrollo infantil. Es por esto queresulta entonces relevante pensar en incorporar a los hombres en los controles perinatales, tanto en talleres psicoeducativos como en el screening universal de sintomatología depresiva, de manera tal de poder detectar y derivar de forma oportuna. Asimismo se hace necesario continuar con campañas de psicoeduación en depresión, como las del Instituto Milenio para el Estudio de la Depresión y la Personalidad (Midap) para desmitificar los conceptos que asocian a la depresión a sus manifestaciones más extremas.

A partir de lo anterior, podríamos inferir que durante el primer año de vida del bebé se va instalando un patrón relacional al interior de la familia, marcado por los roles de género tradicionales, los cuales parecieran ser una repetición de modelos conocidos, mandatos culturales y condiciones estructurales, más que estar basados en una reflexión y acuerdo conjunto de la pareja. El malestar asociado a esta rigidización en los roles y en las narrativas, tampoco es elaborado al interior de la pareja, sino que, en el caso de los hombres, se canaliza muchas veces a partir de la evasión. La posibilidad de diálogo y de conectarse con la vulnerabilidad prácticamente desaparece en este modelo de relación y en las narrativas imperantes, donde se espera que el hombre debe ser capaz de salir solo de sus problemas.

Frente a este escenario, es importante que repensemos la importancia de la coparentalidad, es decir, la capacidad de la pareja de funcionar como equipo en lo que respecta a la crianza, pensando en la necesidad de contar con espacios de diálogo y acuerdo, de manera tal que las decisiones que se vayan tomando al interior de la vida familiar puedan ser vividas como una co-construcción en la que se consideran las perspectivas de sus protagonistas (padres, madres e hijos/as).

Muchas veces, sobre todo en parejas donde la paternidad/ maternidad no se planifica y es vivida como una irrupción en la vida, la posibilidad de reflexionar, de anteponerse a escenarios y de dialogar no se logra gene- 
rar, sino que más bien se reacciona "haciendo lo que hay que hacer”. Es así como la posibilidad de pensar y construir algo singular y propio se esfumina, permitiendo así entonces continuar en una lógica de la disociación, donde la posibilidad de tramitar y elaborar las experiencias no se instala. De esta forma el malestar tampoco se registra a nivel consciente, simplemente se padece con efectos a nivel individual y familiar.

Sin duda como sociedad tenemos que seguir pensando en como generar las condiciones estructurales que favorezcan una paternidad más consciente, más reflexiva, más libre y más satisfactoria. Son muchas las dimensiones que se atraviesan, legislaciones, beneficios, programas educativos y de salud, condiciones laborales, etc., por lo que el trabajo debe ser pensando interdisciplinariamente. En lo inmediato, los profesionales y equipos que se encuentran en contacto directo con las familias, pueden tener un rol activo en la promoción de la corresponsabilidad en la crianza, dejando de considerar naturalmente a la madre como la cuidadora principal y al padre como el secundario. 


\section{Bibliografía}

A Faisal-Cury, A., \& Menezes, P. R. (2007). Prevalence of anxiety and depression during pregnancy in a private setting sample. Archives of Women's Mental Health,, 10, 25-32. doi:10.1007/s00737-006-0164-6

Alvarado, R., Guajardo, V., Rojas, G., \& Jadresic, E. (2012). Validación de la Escala Edimburgo para Embarazadas.web.minsal.cl/sites/default/ files/files/InformeFinalproyectovalidacionEPDSembarazadasV2.pdf: Ministerio de Salud.

Alvarado, R., Rojas, M., Monardes, J., Perucca, E., E Neves, Olea, E., \& Vera, A. (2000). Cuadros depresivos en el postparto en una cohorte de embarazadas: construcción de un modelo causal. Revista chilena de neuro-psiquiatría, 38(2), 84-93.

Baldwin, S., \& Kelly, P. (2015). Postnatal Depression: Don’t reinvented the wheel. Community Practitioner, 88(9), 37-40.

Beck, A., Ward, C., Meldelson, M., Mock, J., \& Erlbaugh, J. (1961). An inventory for measuring depression. Archives of General Psychiatry 4, 561-571. doi:10.1001/archpsyc.1961.01710120031004

Casas, L., \& Valenzuela, E. (2012). Proteccioìn a la maternidad: una historia de tensiones entre los derechos de infancia y los derechos de las trabajadoras. Revista de Derecho, $X X V(1), 77-101$.

Cierpka, M., Scholtes, K., Frey, B., \& Köhler, H. (2011). Der Übergang zur Elternschaft. In K. H (Ed.), Handbuch der Kleinkindforschung. Bern: Huber.

Condon, J., Boyce, P., \& Corkindale, C. (2004). The First-Time Fathers Study: a prospective study of the mental health and wellbeing of men during the transition to parenthood. Australian and New Zealand Journal of Psychiatry, 38(1-2), 56-64.

Cornejo, M., Besoain, C., \& Mendoza, F. (2011). Desafíos en la generación de conocimiento en la investigación social cualitativa contemporánea. Forum: Qualitative Social Research, 12(1).

Cox, J. (2005). Postnatal depression in fathers. Lancet, 366.

Cox, J., Holden, J., \& Sagovsky, R. (1987). Detection of postnatal depression. Development of the 10-item Edinburgh Postnatal Depression Scale. The British Journal of Psychiatry, 150, 782-786.

Coyl, D., Roggman, L., \& Newland, L. (2002). Stress, maternal depression, and negative mother-infant interactions in relation to infant 
attachment. Infant Mental Health Journal, 23(1-2), 145-163. doi:10.1002/imhj.10009

Eberhard-Gran, M., Eskild, A., Tambs, K., Opiordsmoen, S., \& Samuelsen, O. (2001). Review of validation studies of the Edinburgh Postnatal Depression Scale. Acta Psychiatr Scand, 104, 243-249.

Edmonson, O., Psychogiou, L., Vlachos, H., Netsi, E., \& Ramchandani, P. (2010). Depression in fathers in the postnatal period: Assessment of the Edimburgh Postnatal Depression Scale as a screening measure. Journal of Affective Disorders, 125, 365-368. doi:10.1016/ j.jad.2010.01.069

Epifanio, M., Genna, V., Luca, C. D., Roccella, M., \& Grutta, S. L. (2015). Paternal and Maternal Transition to Parenthood: The Risk of Postpartum Depression and Parenting Stress. Pediatr Rep, 7(2). doi:10.4081/pr.2015.5872

Escribe-Agüir, V., Gonzalez-Galarzo, M. C., Barona-Vilar, C., \& Artazcoz, L. (2008). Factors related to depression during pregnancy: Are these gender differences? Epidemiology and Commmunity Health, 62, 410414. doi:10.1136/ jech.2007.063016.

Field, T. (2011). Prenatal depression effects on development. Infant Behavior and Development, 24, 1-14. doi:10.1016/j.infbeh.2010.09.008

Frevert, G., Cierpka, M., \& Joraschky, P. (2008). Familiäre Lebenszyklen. . In M. Cierpka (Ed.), Handbuch der Familiendiagnostik (3 ed.). Heidelberg: Springer.

Gavin, N. I., Gaynes, B. N., Lohr, K. N., Meltzer-Brody, S., Gartlehner, G., \& Swinson, T. (2005). Perinatal depression: A systematic review of prevalence and incidence. Obstetrics and Gynecology, 106, 1071-1083.

Goodman, J. (2004). Paternal postpartum depression, its relationship to maternal postpartum depression,and implications for family health. J Adv Nurs, 45(1), 26-35.

Goodman, S., Rouse, M., Conell, A., Broth, M. R., Hall, A., \& Heyward, D. (2011). Maternal Depression and Child Psychopathology: A MetaAnalytic Review. Clinical Child Family Psychology Review, 14(1), 127. doi:10.1007/s10567-010-0080-1

Herrera, F., \& Pavicevic, Y. (2016). Anticipando la Paternidad: Ella es la que está embarazada. Masculinities and Social Change, 5(2), 107-133. doi:10.17583/MCS.2016.2038

Jadresic, E. (2010). Depresión en el embarazo y el puerperio. Revista chilena de neuro-psiquiatría, 48(4), 269-278. 
Jadresic, E., Araya, R., \& Jara, C. (1995). Validation of the Edinburgh Postnatal Depression Scale (EPDS) in Chilean postpartum women. $J$ Psychosom Obstet Gynaecol, 16(4), 187-191.

Jadresic, E., Nguyen, D., \& Halbreich, U. (2007). What does Chilean research tell us about postpartum depression? Journal of Affective Disorders, 102, 237-243.

Jones, E., \& Asen, E. (2004). Terapia sistémica de pareja y depresión. Bilbao, España: Desclée De Brouwer.

King, V. (2010). Bedingungen der ElternschaftskonstellationUmgestaltungen der Identität von der Adoleszenz zu väterlichen und mütterlichen Kompetenzen. Kinderanalyse, 18(1), 1-27.

Lai, B., Tang, A., Lee, D., Yip, A., \& Chung, T. (2010). Detecting postnatal depression in Chinese men: A comparison of three instruments.Psychiatry Research, 180, 80-85. doi:10.1016/ j.psychres.2009.07.015

Mason, J. (2002). Qualitative researching. London Thousand Oaks New Dehli: SAGE.

Massoudi, P., Hwang, C., \& Wickberg, B. (2013). How well does the Edinburgh Postnatal Depression Scale identify depression and anxiety in fathers? A validation study in a population based Swedish sample. . Journal of Affective Disorders, 149, 67-74. doi:10.1016/ j.jad.2013.01.005

Matthey, S., Barnett, B., Kavanagh, D., \& Howie, P. (2001). Validation of the Edinburgh Postnatal Depression Scale for men, and comparison of item endorsement with their partners. Journal of Affective Disorders, 64(2-3), 175-184.

Miller, T. (2017). Who is balancing caring and paid work in the UK? Paper presented at the Proveer, cuidar y criar: discursos y experiencias de los 'nuevos padres'.Santiago, Chile: Universidad Diego Portales

O’Hara, M. (2009). Postpartum depression: what we know. J Clin Psychol, 65, 1258-1269.

Olavarria, J., \& Marquéz, F. (2004). Varones, entre lo Público y la Intimidad.

Paulson, J., \& Bazemore, S. (2010). Prenatal and Postpartum Depression in Fathers and Its Association With Maternal Depression A Metaanalysis. JAMA, 303(10). 
Paulson, J., Bazemore, S., Goodman, J., \& Leiferman, J. (2016). The course and interrelationship of maternal and paternal perinatal depression. Archives of Women's Mental Health, 1-9.

Paulson, J., Keefe, H., \& Leiferman, J. (2009). Early parental depression and child language development. J Child Psychol Psychiatry, 50(3), 254-262.

Pérez, F., Brahm, P., Riquelme, S., Rivera, C., Jaramillo, K., \& Eickhorst, A. (2017). Paternal post-partum depression: How has it been assessed? A literature review. Universidad Alberto Hurtado. Artículo no publicado.

Pérez, F., Catalán, A., Morales, A., Quinlan, A., Riquelme, D., Navarro, J., \& Valdés, C. (2017). Assessment of Postpartum Depression in a Group of Chilean Parents. Universidad Alberto Hurtado. Artículo no publicado.

Ramchandani, P., Stein, A., Evans, J., O’Connor, T., \& Team, A. S. (2005). Paternal depression in thepostnatal period and child development: a prospective population study. Lancet, 365(9478), 2201- 2205.

Stern, D. (1998). The Motherhood Constellation: A Unified View of Parent Infant Psychotherapy London: Karnac Books.

Stern, D., Bruschweiler-Stern, N., \& Freeland, A. (1998). The birth of a mother. New York, EUA: Basic Books.

Tran, T., Tran, T., \& Fisher, J. (2012). Validation of three psychometric instruments for screening for perinatal common mental disorders in men in the north of Vietnam. . Journal of Affective Disorders, 136, 104-109. doi:10.1016/j.jad.2011.08.012

Valdés, C., Morales-Reyes, I., Pérez, C., Medellín, A., Rojas, G., \& Krause, M. (2017). Propiedades psicométricas del Inventario de Depresión de Beck para la población chilena. Revista Médica de Chile, 145(8), 1005-1012. doi:10.4067/s0034-98872017000801005

Valdés, X., \& Godoy, C. (2008). El lugar del padre: rupturas y herencias. Representaciones de la paternidad en grupos altos, medios y populares chilenos. Estudios Avanzados, 6(9), 79-112.

Vázquez, C., \& Sanz, J. (1999). Fiabilidad y validez de la versión española del Inventario para la Depresión de Beck de 1978 en pacientes con trastornos psicológicos. Clínica y Salud, 10(1), 59-81.

Veskrna, L. (2013). Peripartum depression - does it occur in fathers and does it matter? Journal of Men's Health., 7(4), 420-430. doi:10.1016/ j.jomh.2010.10.004. 
WHO. (2016). Maternal and child mental health.

Beck, A., Ward, C., Meldelson, M., Mock, J., \& Erlbaugh, J. (1961). An inventory for measuring depression. Archives of General Psychiatry 4, 561-571. doi: 10.1001/archpsyc.1961.01710120031004

Recibido: 30.04 .18

Aceptado: 31.07 .18 